\title{
Effective New Plasma Cleaning Strategies for Scanning Electron Microscopes and FIBs
}

\author{
Ronald Vane ${ }^{1}$ and Michael Cable ${ }^{1}$ \\ ${ }^{1}$ XEI Scientific, Redwood City, CA 94063
}

Remote plasma cleaning can be performed on SEMs and FIBs at pressures below 100 mTorr (13.3 Pa) during direct pumping with a TMP (Turbo Molecular Pump). Previous studies ${ }^{1}$ showed that low chamber pressures increase the rate of cleaning and the distances at which cleaning was observed. If the chamber pressure can be brought down to $<25 \mathrm{mT}$ Torr and the flow of plasma cleaning gas into the chamber down to $<25 \mathrm{sccm}$ then using the Evactron ${ }^{\circledR}$ remote plasma cleaners results in flowing afterglow cleaning ${ }^{2}$. With $20 \mathrm{sccm}$ of air flow rate, cleaning rates are as much as $100 \mathrm{X}$ faster than those above 400 mTorr, and there is the ability to clean larger volumes. Most TMPs can be operated without overheating, but active cooling may be needed on some TMP models at higher flow rates. Low pressures increase cleaning speeds by increasing mean free paths and reducing the recombination rates of the oxygen radicals by three body collisions. Achieving lower pressures by reducing flow rates below 10 sccm starves the plasma of the gas needed to produce cleaning radicals. Plasmas operating below 1 $\mathrm{mTorr}$ in the chamber can be achieved, but if the pressure is $10^{-5}$ Torr, the concentration of $\mathrm{O}$ radicals is 0.01 of that produced at 1.0 mTorr. This remarkable result plus new plasma ignition methods at low pressures allow more effective cleaning strategies for large instrument vacuum chambers.

XEI Scientific has developed a new plasma ignition technology, "Pop" ignition, that allows Evactron plasma cleaning to be started directly from very high vacuum chamber pressure $(<1 \mathrm{mTorr})$ and then to operate in the 1 to 20 mTorr range with full speed turbo pump operation. The needed pressure changes are part of the ignition process and the evacuation controls of the instrument are untouched. Cleaning is very fast at this pressure as illustrated in Figures 1 and 2. Figure is an RGA spectrum taken on a 48 liter cylindrical chamber that has been contaminated by pumping on petroleum jelly overnight. It shows a typical hydrocarbon mass spectrum with a series of peaks 14 amu apart. In figure 2 the chamber has been cleaned with 2 one minute plasma cleans and all of the hydrocarbon signature peaks have been removed leaving only greatly reduced water and air peaks.

Before this new technology was developed, classic Evactron plasma ignition and cleaning was done at roughing pump pressures. This was done to assure no damage to electron and ion guns because the gun valves would be closed and to avoid pumping through diffusion pumps. Because plasma cleaning was slow at these pressures some users cleaned for long periods to assure cleaning of large or very contaminated chambers. With the new technology short cleaning times of under 5 minutes are often sufficient to remove all carbon. This allows overnight cleaning to be avoided, and it is discouraged because of extended exposure of the instrument interior to $\mathrm{O}$ radicals.

The "pop" ignition also allows the plasma to be turned off after cleaning a short (1-5 minutes) time, allowing a quick return to base pressure with the turbo pump to remove reaction products, and then restarting the plasma to repeat the cleaning cycle. While at base pressure any hidden hydrocarbons can degas and redistribute within the chamber due to long free path molecular flow where they can be removed in the next plasma cycle. Cycle plasma cleaning is shown to be a very effective way to get the chamber pristine using this new plasma cleaning technology at high vacuum. 
References:

1. C.A. Morgan and R. Vane "Carbon Contamination removal in larger chambers with low-power plasma cleaning” Proceedings of SPIE, vol 8324, 9 March 2012 \, pages 83242F-83242F-8, XP055160546, ISSN: 0277-786X, DOI: 10.1117/12.917786

2. Ronald Vane et al. "Advancements in Decontamination of Vacuum Systems Using Plasma Cleaning” Micrcoscopy and Microanalysis 2104 poster 566

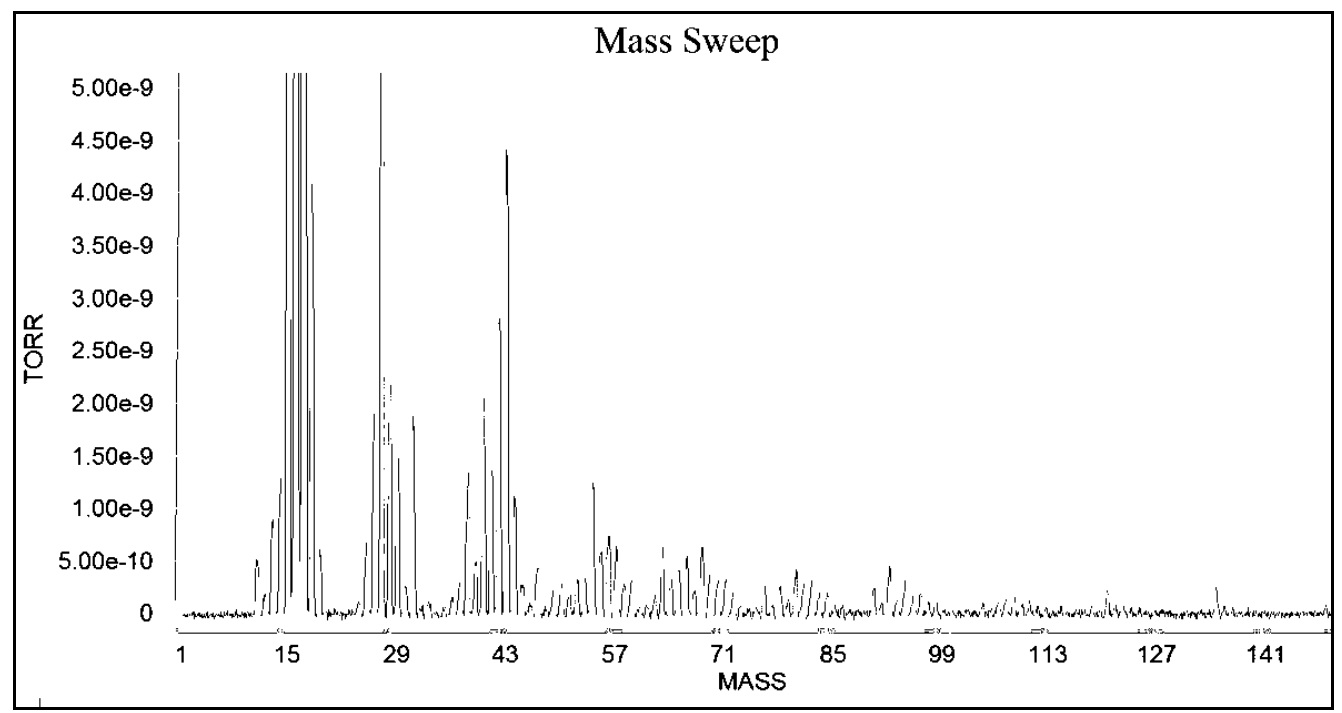

Fig. 1 RGA mass spectrum of evaporated petroleum jelly (Vaseline) contamination. Series of peaks at show hydrocarbons

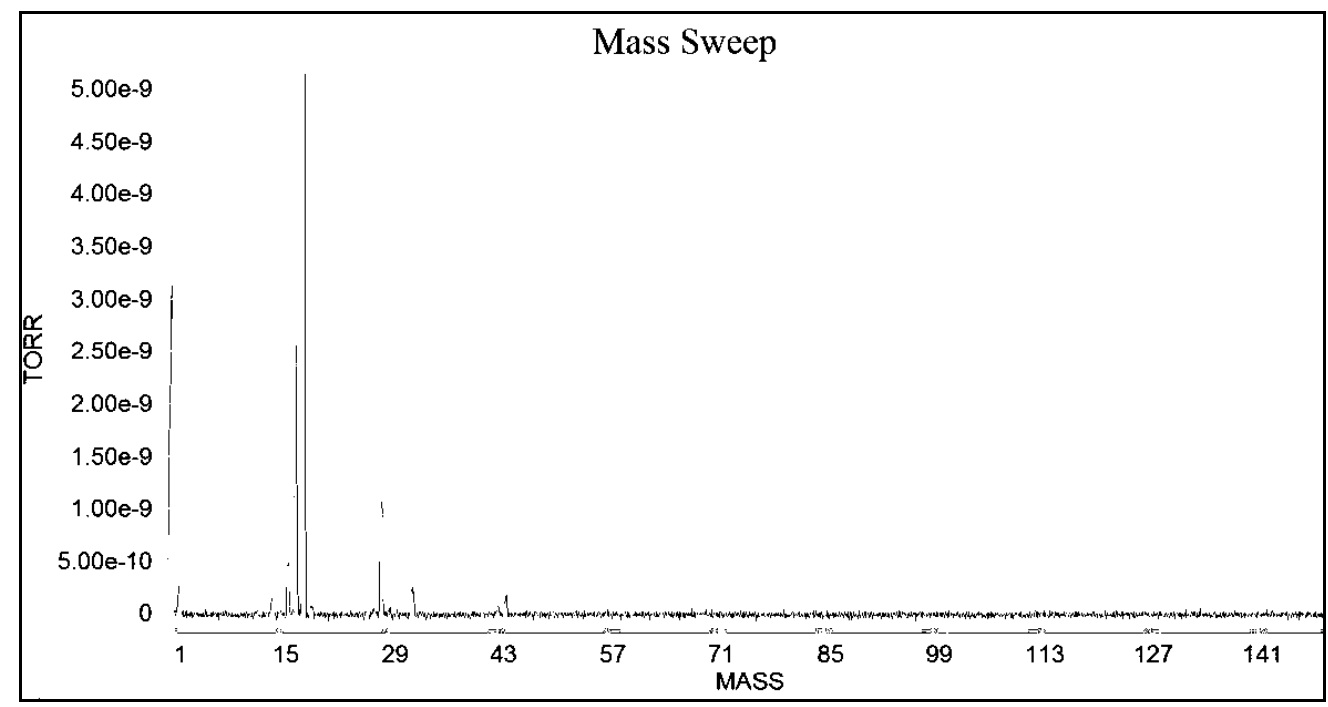

Fig. 2 RGA mass spectrum at base pressure after two 1 minute cleanings with the Evactron ES model at 20W and 2 mTorr pressure in Chamber. Remaining peaks are from air and water. 